\title{
Processo de cuidar nas instituições de longa permanência: visão dos cuidadores formais de idosos
}

\author{
Care process in long term care facilities: elderly caregivers' views
}

Proceso de atención en hogares para ancianos: la visión de los cuidadores

\section{Marco Túlio de Freitas Ribeiro', RaQuel Conceição Ferreira', Cláudia Silami de Magalhães', Allyson Nogueira Moreira', Efigênia Ferreira e Ferreira'}

'Universidade Federal de Minas Gerais. Departamento de Odontologia Social e Preventiva. Belo Horizonte, MG

Submissão: $12 / 12 / 2008$

Aprovação: 10/08/2009

\section{RESUMO}

Este estudo avaliou o cuidar nas instituições de longa permanência filantrópicas e privadas de Belo Horizonte, Brasil. Uma amostra de 181 cuidadores de idosos foi entrevistada. Avaliou-se razão idosos/cuidador, sua formação em serviço, satisfação, dificuldades na função e atividades executadas. A razão idosos/cuidador foi maior nas filantrópicas. A maioria adouiriu suas habilidades no auxílio aos idosos da própria instituição. Nas privadas, maior percentual aprendeu suas habilidades em cursos e 54,7\% realizaram treinamento após admissão, percentual maior nas privadas $(64,6 \%, \mathrm{p}<0,05)$. Quase todos relataram satisfação na profissão, porém $51,4 \%$ afirmaram enfrentar dificuldades na prática. A atividade mais realizada foi banho dos idosos $(22,5 \%)$. Foram apontados problemas Quanto à estrutura e a prestação de serviços a idosos nas instituições, principalmente nas filantrópicas.

Descritores: Cuidadores; Instituição de longa permanência para idosos; Assistência a idosos.

ABSTRACT

This study evaluated the care in philanthropic and private long-term institutions in Belo Horizonte, Brazil. A sample of I8I caregivers of elderly was interviewed and was evaluated rate elderly / caregiver, their training in service, satisfaction, difficulties in function and activities executed. The rate elderly / caregiver was higher in philanthropic. Most aceuired their duties in helping the elderly. In private, a higher percentage learned their duties in courses and $54.7 \%$ have training after admission, the percentage being higher in private institutions (64.6\%, $\mathrm{p}<0.05)$. Almost all reported satisfaction in the profession, but $51.4 \%$ reported difficulties in practice. The activity carried out more was the bath of the elderly(22.5\%). Problems were raised regarding the structure and provision of services to elderly in institutions, especially in philanthropic.

Descriptors: Caregivers; Homes for the aged; Old age assistance.

\section{RESUMEN}

Este studio evaluó el cuidar en los hogares de los ancianos filantrópicos y privados de Belo Horizonte, Brasil entrevistando I 81 cuidadores. Se evaluó la razón ancianos/cuidadores, su formación en trabajo, satisfación y dificuldades en sus funciones. La razón ancianos/cuidador fue más grande en las filantrópicas. La mayoría adeuirio las habilidades en el auxilio de los ancianos de la institución. En las privadas, mayor percentual aprendió sus habilidades en cursos y 54,7\% realizaron pues admisión, porcetaje mayor en las privadas (64,6\%, $\mathrm{p}<0,05)$. Casi todos relataron satisfación. Ellos afirmaron tener dificultad en la práctica $(51,4 \%)$. La actividad más realizada fue el baño $(22,5 \%)$. Fueron detectados problemas en la estructura y la prestación de servicios a los ancianos, principalmente en las institutiones filantrópicas.

Descritores: Cuidadores; Hogares para Ancianos; Asistencia a los ancianos. 


\section{INTRODUÇÃO}

Questões como os cuidados a idosos institucionalizados têm despertado a preocupação de vários setores da sociedade devido ao crescente aumento na proporção de idosos na população. O interesse em torno do cuidado ao idoso institucionalizado no Brasil reflete o momento atual da transição demográfica, onde de um lado há um aumento na demanda por instituições, e de outro surgem denúncias Quanto à precariedade das condições físicas de algumas instituições asilares brasileiras, assim como das condições desumanas e maus tratos a Que os idosos são submetidos em algumas destas ${ }^{(1,2)}$.

Dentro deste contexto, é propício resgatar o significado original da palavra asilo, Que se origina do grego (asylum), referindo-se a um local de amparo e proteção. Politicamente, esta palavra descreve a acolhida Que determinados países dão a pessoas perseguidas politicamente confirmando o sentido de acolhimento ao Qual se associa. Asilo é utilizado ainda para descrever instituições de assistência social cuja finalidade é abrigar idosos pobres, tendo uma conotação fortemente depreciativa. Com o aumento na demanda por instituições de longa permanência, devido às mudanças demográficas e familiares, a partir da década de 70 do século XX, começaram a surgir instituições privadas para idosos, de fins lucrativos, as Quais adotam terminologias diferentes, tais como: casas de repouso e clínicas geriátricas. Assim, os asilos, passaram a incorporar outros extratos sociais Que não somente os pobres ${ }^{(3)}$.

Depreende se que ao longo do tempo, o sentido de amparo e proteção do "asylum" originalmente atribuído ao termo foi substituído pelo aspecto assistencial. A partir do momento que os asilos passaram a constituir uma demanda de extratos sociais de maior poder aquisitivo, constituindo um novo mercado de trabalho, uma nova terminologia foi adotada no sentido de diferenciar as instituições destinadas aos idosos pobres dos ricos. Entretanto, somente uma mudança de nomes não é suficiente para romper com o estigma em torno dos asilos e da institucionalização de idosos. De forma geral, os termos adotados convergem para denominações como de "abrigo" e "asilo", que definem um conjunto de equipamentos sociais, públicos, confessionais ou privados aos Quais faltam normas, padrões e, sobretudo, respeito à subjetividade dos velhos Que abrigam ${ }^{(1)}$. Portanto, Quer seja uma instituição filantrópica, ou uma instituição de caráter privado, o centro da discussão em relação a tais locais deve ser Quanto ao tipo de cuidado prestado aos seus residentes.

Dentro de uma abordagem de vigilância para promoção da saúde, Que considera o processo saúde-doença-cuidado através de um enfoque técnico e político ${ }^{(4,5)}$, o cuidado ao idoso institucionalizado demanda uma atenção contínua para Que nenhum aspecto seja negligenciado. No Brasil, a avaliação da Qualidade dos cuidados oferecidos nas instituições de longa permanência brasileiras não é feita de forma sistemática apontando resultados conflitantes ${ }^{(6)}$. Qualidade do cuidado é um termo de difícil definição e se refere a julgamentos de valores aplicados a determinados aspectos, propriedades, ingredientes ou dimensões do cuidado ${ }^{(7)}$. Em função da dificuldade de se avaliar o processo (cuidado) nas instituições de longa permanência, alguns autores determinaram a Qualidade da atenção prestada aos idosos nestes locais a partir da estrutura das mesmas ${ }^{(6)}$.
Do exposto acima, depreende-se Que os cuidadores formais, profissionais contratados para auxiliar os idosos em suas atividades básicas e instrumentais de vida diária ${ }^{(8)}$, são de fundamental importância nestes espaços. O cuidado dispensado ao idoso transforma-se, no geral, em uma tarefa árdua e complexa. Muitas vezes, tal atividade gera sentimentos de angústia, insegurança e desânimo. Esse processo, entretanto, varia de pessoa a pessoa e não ocorre com todos os cuidadores. Alguns deles podem, inclusive, sentir prazer e conforto, Quando conseguem bons resultados, independentemente dos esforços físicos e psíquicos exigidos. Muitos sentimentos se sobrepõem na relação cuidador-idoso, cuidadorfamiliares. $\mathrm{O}$ fato de não conseguir lidar com as dificuldades pode levar o idoso a comportamentos incompreensíveis, incomodando particularmente ao cuidador. Assim, além de saber lidar com a doença, o cuidador tem Que conviver com a subjetividade inerente às relações humanas ${ }^{(9)}$. $\mathrm{O}$ relato dos atores centrais do processo de cuidar - cuidadores - deve ser considerado Quando na avaliação da Qualidade da assistência prestada ao idoso nas instituições de longa permanência.

Diante das considerações apresentadas, o objetivo deste trabalho foi avaliar o cuidar nas instituições de longa permanência considerando não somente a sua estrutura, mas também os relatos dos cuidadores de idosos.

\section{METODOLOGIA}

Este é um estudo observacional, do tipo transversal realizado com os cuidadores de idosos das instituições de longa permanência filantrópicas e privadas de Belo Horizonte, Brasil.

Para a realização deste trabalho foi estabelecida uma parceria entre a Promotoria de Justiça de Defesa da Pessoa Portadora de Deficiência e Idosos e o Colegiado de Pós Graduação da Faculdade de Odontologia da Universidade de Minas Gerais, através do credenciamento do pesquisador como voluntário da primeira. O vínculo entre essas duas entidades possibilitou ao pesquisador o acesso aos dados cadastrais das instituições de Belo Horizonte e a realização do censo dos cuidadores Que trabalhavam nas mesmas.

O universo considerado para este estudo foi de 512 cuidadores formais, de Belo Horizonte. Deste total, 310 cuidadores trabalhavam em instituições definidas pela Promotoria de Justiça de Defesa da Pessoa Portadora de Deficiência e Idosos como filantrópicas, Que possuem entidade mantenedora, e 202 exerciam suas atividades em instituições privadas, Que cobram mensalidade de seus residentes.

A amostra foi estimada por meio do método de comparação entre proporções, considerando-se um nível de significância de $95 \%(a=0,05)$ e um poder de teste de $90 \%(b=0,10)$. Os valores considerados para comparação foram os percentuais de cuidadores nas instituições filantrópicas $(60,5 \%)$ e privadas $(39,5 \%)$. Após a correção para população finita obteve-se uma amostra de 181 cuidadores (98 em filantrópicas e 83 em privadas). Os cuidadores foram selecionados aleatoriamente por sorteio sistemático a partir da listagem de cuidadores de cada instituição.

A coleta de dados foi realizada por meio de entrevistas, feitas por um único pesquisador, seguindo roteiro estruturado previamente testado. As características estruturais da instituição foram avaliadas considerando as variáveis: razão de idosos por cuidador, a formação 
dos cuidadores em serviço, sua satisfação, dificuldades com a função e atividades executadas. Para uma análise em profundidade do cuidado, foram feitas perguntas abertas aos cuidadores de idosos das instituições de longa permanência de Belo Horizonte.

Os dados foram agrupados em categorias de análise e apurados em uma planilha do programa Microsoft Excel. A comparação entre instituições privadas e filantrópicas foi realizada empregando os testes Qui-Quadrado ou exato de Fisher, no programa Epiinfo versão 3.4. Foram selecionados relatos considerados representativos para apresentação dos resultados. O projeto foi aprovado pelo Comitê de Ética em Pesquisa da Universidade Federal de Minas Gerais (Parecer 212/01). Todos os participantes do estudo e os coordenadores das instituições de longa permanência assinaram o Termo de Consentimento Livre e Esclarecido concordando em participar da peseuisa.

\section{RESULTADOS E DISCUSSÃO}

O perfil dos cuidadores de idosos em instituições de longa permanência de Belo Horizonte é representado, na sua maioria, por profissionais do sexo feminino, com estado civil solteiro ou casado, com menos de 50 anos de idade, de classe econômica C, com remuneração mensal de menos de 2 salários mínimos, Que trabalham nas instituições e atuam nesta profissão há menos de 2 anos. Nas instituições privadas, o número de cuidadores com mais de 5 anos de estudos é significativamente maior Que nas filantrópicas $(p=0,00)$. O perfil desses profissionais foi descrito e discutido detalhadamente em trabalho anterior ${ }^{(10)}$.

No presente estudo, a razão de idosos por cuidador foi maior nas instituições filantrópicas $(3,6)$ Que nas privadas $(2,4)$. Em percentuais, estes dados mostraram Que os cuidadores das instituições filantrópicas auxiliavam 33,3\% mais idosos Que os das instituições privadas.

Relatos de cuidadores de instituições filantrópicas indicam Que nesta modalidade de instituição, os profissionais têm pouca disponibilidade para os residentes: "Queria ter mais tempo para me dedicar a eles... mas tenho de fazer outras coisas"; "... falta de tempo"

A maior parte dos indivíduos avaliados relatou a aquisição de suas habilidades profissionais no auxílio diário aos idosos da própria instituição (Tabela 1). Esse achado vai ao encontro das descrições de Duarte ${ }^{(8)}$, segundo as Quais muitos daqueles que se dispõe a exercer a função de cuidadores de idosos não possuem capacitação para tal. Os relatos de muitos dos cuidadores sobre sua inserção na profissão reforçam esta observação. "Eles Quiseram me colocar de cuidador... eu estava trabalhando na obra daqui"

Entretanto, nas instituições privadas, um maior percentual de cuidadores relatou ter aprendido suas habilidades por meio de cursos técnicos (Tabela 1), o Que sugere uma tendência de melhor formação dos profissionais das instituições privadas. A importância da formação de cuidadores de idosos é amplamente discutida na literatura, pois, o cuidado dispensado ao idoso é influenciado por valores, crenças e experiências vividas ${ }^{(1)}$. Por mais Que o cuidado ao idoso seja ontológico, ele demanda especificidades que precisam ser consideradas a luz do exercício profissional, sob o risco de geração de iatrogenias (12). Portanto, a formação dos cuidadores constitui um importante indicador a ser observado na avaliação das instituições de longa permanência.

A promoção de funcionários contratados para serviços gerais à função de cuidador foi observada, principalmente, em instituições filantrópicas, confirmando uma menor Qualificação dos profissionais desta modalidade. Ribeiro et al. ${ }^{(10)}$ observaram Que a formação técnica dos funcionários não é priorizada na contratação dos cuidadores das instituições filantrópicas. De acordo com os autores,

Tabela 1. Ccomparação entre as modalidades das instituições de longa permanência Quanto ao tipo de formação profissional, formação em serviço, satisfação e dificuldades na profissão. Belo Horizonte, 2006.

\begin{tabular}{|c|c|c|c|c|c|c|c|}
\hline \multirow{3}{*}{\multicolumn{2}{|c|}{ Variáveis avaliadas }} & \multicolumn{4}{|c|}{ Modalidades das instituições de longa permanência } & & \\
\hline & & \multicolumn{2}{|c|}{ Filantrópicas } & \multicolumn{2}{|c|}{ Privadas } & \multicolumn{2}{|c|}{ Total } \\
\hline & & $\mathrm{n}$ & $\%$ & $\mathrm{n}$ & $\%$ & $\mathrm{n}$ & $\%$ \\
\hline \multirow{3}{*}{$\begin{array}{l}\text { Tipo de formação } \\
\text { profissional* }\end{array}$} & Prática na instituição & 52 & 53,0 & 35 & 42,7 & 87 & 48,3 \\
\hline & Curso técnico & 23 & 23,5 & 29 & 35,4 & 52 & 28,9 \\
\hline & $\begin{array}{l}\text { Experiência no cuidado a } \\
\text { familiares }\end{array}$ & 23 & 23,5 & 18 & 21,9 & 41 & 22,8 \\
\hline \multicolumn{8}{|c|}{$p=0,20$} \\
\hline \multirow{2}{*}{ Formação em serviço** } & $\mathrm{Sim}$ & 45 & 46,4 & 53 & 64,6 & 98 & 54,7 \\
\hline & Não & 52 & 53,6 & 29 & 35,4 & 81 & 45,3 \\
\hline \multicolumn{8}{|c|}{$p=0,01$} \\
\hline \multirow{2}{*}{ Satisfação com a profissão } & Sim & 95 & 96,9 & 82 & 98,8 & 177 & 97,8 \\
\hline & Não & 3 & 3,1 & 1 & 1,2 & 4 & 2,2 \\
\hline \multicolumn{8}{|c|}{$p=0,38$} \\
\hline \multirow{2}{*}{ Dificuldades na prática } & Sim & 50 & 51,0 & 43 & 51,8 & 93 & 51,4 \\
\hline & Não & 48 & 49,0 & 40 & 48,2 & 88 & 48,6 \\
\hline \multicolumn{8}{|c|}{$p=0,97$} \\
\hline
\end{tabular}

* Um Questionário sem resposta de um cuidador de instituição privada

**Um questionário sem resposta em cada modalidade

***Comparação entre modalidades das instituiçôes realizada pelo teste Qui-Quadrado e Exato de Fisher. 
o fato de estas instituições possuírem entidades mantenedoras religiosas determina um processo seletivo de funcionários regido por valores diferentes dos adotados pelas de caráter privado. Além disto, os cuidadores de idosos das instituições filantrópicas possuem baixa escolaridade e menor acesso à informação Quando comparado aos de instituições privadas ${ }^{(10)}$. Estes resultados mostram a necessidade de programas de capacitação profissional para cuidadores de idosos, especialmente nas instituições filantrópicas, onde profissionais de baixa escolaridade e sem formação técnica são alocados nesta função.

A formação em serviço foi avaliada pelo Questionamento dos cuidadores sobre a realização de treinamento após sua admissão, com $54,7 \%$ de respostas afirmativas. Nas instituições privadas esse tipo de capacitação foi mais freeüente Que nas instituições (Tabela I).

Nas instituições filantrópicas, ao contrário das privadas, as atividades de treinamento de cuidadores não são uma rotina, contrariando as determinações da Anvisa ${ }^{(12)}$. De acordo com este órgão regulamentador do funcionamento das instituições de longa permanência, estas deverão realizar atividades de educação permanente na área de gerontologia com a finalidade de aprimorar tecnicamente os recursos humanos envolvidos na prestação de serviços aos idosos. Considerando a menor formação técnica e escolaridade $^{(10)}$ dos cuidadores das instituições filantrópicas, a incorporação de profissionais de outras áreas (faxina e serviços gerais) a esta atividade, a elaboração/execução de atividades de educação permanente depende de uma integração intersetorial. Em muitas instituições, em especial as de caráter filantrópico, profissionais sem formação adequada tendem a reproduzir procedimentos Que anulam a subjetividade dos idosos, reproduzindo uma cultura de pessoas Que criam e ao mesmo tempo são vítimas delas. Desta forma, ações conjuntas da Anvisa com os pólos de formação de recursos humanos devem ser direcionadas a educação permanente dos cuidadores de idosos das instituições de longa permanência.

É importante frisar Que, mesmo os profissionais com curso superior ou técnico na área de enfermagem, descreveram a importância do trabalho na instituição em sua formação, ou seja, o ensino formal não lhes forneceu o embasamento suficiente para sua prática. Esse resultado sugere a necessidade de reestruturação do estágio profissional nos cursos de formação dos cuidadores. Portanto, a formação em serviço destaca-se como uma importante alternativa para Qualificação dos cuidadores de idosos, principalmente ao se considerar a forma de inserção destes profissionais nas instituições, especialmente nas filantrópicas. Além disso, a capacitação dos cuidadores de idosos constitui uma das diretrizes das políticas de atenção ao idoso ${ }^{(14,15)}$.

A maioria dos cuidadores, tanto nas instituições filantrópicas como privadas, relatou satisfação com a profissão (Tabela 1). Dos cuidadores satisfeitos, 100,0\% nas instituições privadas citaram o "bem estar pessoal" como motivo da satisfação. Entre os cuidadores satisfeitos das instituições filantrópicas $(n=95)$, outros motivos além do bem estar pessoal $(93,7 \%)$, bem menos citados foram: "sustento próprio" (2,0\%) e "oportunidade de emprego" (4,3\%). O alto percentual de satisfação verificado confirma as tendências recentes na pesquisa sobre cuidadores. Ainda Que o cuidar seja uma tarefa complexa, é também fonte de prazer. Teixeira ${ }^{(9)}$ relatou Que, apesar das grandes exigências dessa profissão, cuidar de idosos dependentes pode ser uma fonte de realização pessoal.

Ilustrando o motivo da satisfação com a função, algumas falas apontam para uma identificação entre cuidadores e idosos.

“Eu vejo neles aquilo Que posso precisar amanhã. É gratificante o que se faz por eles"

"Eu gosto de lidar com as idosas,... fico pensando em mim mais tarde."

“Igual a gente vai olhando os avôs da gente...a gente também é igual, eu estou ficando velha..."

"No início achei difícil....depois eu aprendi... é o caminho que você vai passar se não morrer antes"

Muitos cuidadores identificam neles próprios o risco de institucionalização na velhice. A assimilação da condição dos idosos institucionalizados por parte dos cuidadores pode ser mais bem compreendida considerando o perfil destes profissionais. Fatores de risco para institucionalização como: gênero, a solidão, a pobreza são aspectos comuns entre idosos e seus cuidadores. Estes resultados sugerem a necessidade de pesquisas Que avaliem com maior profundidade as implicações da identificação cuidador/idoso no estabelecimento de vínculos de solidariedade entre estes.

Reforçando a descrição anterior Quanto aos cuidadores nas instituições filantrópicas, o discurso de alguns destes mostrou Que o contentamento descrito por eles decorre do fato de terem conseguido, através desta atividade, a possibilidade de inserção no mercado de trabalho.

\section{"Preciso trabalhar"}

"É um trabalho que eu gosto de fazer... é uma oportunidade de estar trabalhando"

"Foi me dada a oportunidade em uma época difícil da minha vida... meu marido estava desempregado"

Cabe, portanto, uma reflexão Quanto à satisfação destes profissionais com seu trabalho, se esta é uma conseQüência da atividade de cuidar ou de ter conseguido o trabalho, mesmo sem formação profissional.

Mesmo satisfeitos com a profissão, mais da metade $(51,4 \%)$ dos cuidadores relatou enfrentar dificuldades na sua prática diária (Tabela I). Em ambas as modalidades das instituições, a dificuldade mais freeüentemente relatada pelos cuidadores foi "de relacionamento com o idoso" seguida por "dificuldades na transferência do idoso". Oito cuidadores das instituições filantrópicas relataram dificuldades Que não se repetiram entre os cuidadores das privadas, tais como: "Abandono dos idosos" (1), "Cansaço e excesso de trabalho" (2), "Falta de remédio na instituição" (1), "Falta de tempo" (1), "Limpeza e lavar roupa" (2) e "Responsabilidade com o dinheiro" (1). Esses dados demonstram a complexidade do cuidar $^{(16)}$.

Como constatado neste trabalho e relatado previamente por trabalhos clássicos da literatura gerontológica ${ }^{(17)}$, problemas de convivência entre idosos institucionalizados são freqüentes. Estudos 
realizados com idosos residentes em instituições de longa permanência descreveram elevados percentuais de idosos institucionalizados etilistas, deprimidos ou demenciados ${ }^{(18-20)}$. Problemas de relacionamento podem gerar situações de violência contra o idoso $^{(21)}$. devido ao stress dos cuidadores. Desta forma, a capacitação dos cuidadores, deve desenvolver neles habilidades para lidar com os problemas de relacionamento.

O percentual de cuidadores Que descreveu dificuldade com a transferência dos idosos foi maior nas instituições filantrópicas Essa é uma atividade que demanda fisicamente dos cuidadores, pois muitos idosos institucionalizados apresentam problemas de mobilidade ${ }^{(18,22)}$. Como apresentado inicialmente, foi observado uma maior razão idosos/ cuidador nas instituições filantrópicas. Assim, pode-se inferir Que nestas, a transferência pode trazer mais dificuldade para os cuidadores, ainda Que numérica. A declaração de uma cuidadora de uma instituição filantrópica aponta para este fato:

“Peso deles, às vezes precisa de mais pessoas para ajudar".

Dificuldades relacionadas à estrutura das instituições ocorreram somente entre os cuidadores de instituições filantrópicas, Que, além de relatos sobre falta de medicamentos, descreveram duplicidade de funções como fazer a limpeza da instituição e lavar a roupa dos idosos. Uma cuidadora de 72 anos de uma instituição filantrópica fez a seguinte descrição:

"Cuidar das roupas... são 36 velhos... cuido da roupa de todos".

Em outra instituição filantrópica uma cuidadora fez a seguinte declaração:

\section{"Quando estou arrumando o banheiro e tenho Que colocar luva para cuidar deles porque eles sujaram".}

Estas declarações indicam Que nas instituições filantrópicas não há uma definição de funções, os profissionais promovidos à condição de cuidador continuam a executar tarefas Que faziam Quando de sua contratação. Segundo a Anvisa ${ }^{(13)}$, as instituições de longa permanência devem ter em seu Quadro funcionários com vínculo formal para limpeza, serviços de lavanderia de acordo com a área e número de idosos da instituição. Nas instituições filantrópicas, a interferência da falta de divisão de funções entre cuidadores e outros profissionais na eualidade do cuidado ficou clara no relato de alguns profissionais:

"Queria ter mais tempo para me dedicar a eles... mas tenho de fazer outras coisas"; "Falta de tempo".

Portanto, a estrutura das instituições de longa permanência têm influência na Qualidade dos serviços prestados por estas ${ }^{(6,7)}$.
As atividades executadas pelos cuidadores diariamente foram: banho $(22,5 \%)$, alimentação $(21,3 \%)$, higiene íntima $(18,2 \%)$, medicação (16,6\%), serviços gerais (8\%), higiene bucal $(7,5 \%)$, escuta $(4,4 \%)$, vestuário e transferência $(1,5 \%)$. O percentual de cuidadores Que relataram realizar serviços gerais foi maior nas instituições filantrópicas $(37,0 \%)$ Que nas privadas (12,0\%), achado Que confirmou as observações anteriores Quanto ao acúmulo de funções dos cuidadores das instituições filantrópicas. Há Que se considerar Que as instituições filantrópicas são mais antigas( ${ }^{(3)}$, e em sua maioria vinculadas a instituições religiosas ${ }^{(23)}$. Assim, ao longo do tempo, estas instituições desenvolveram práticas eminentemente assistencialistas, sem normatização de condutas, seguindo uma lógica estranha à organização do trabalho.

Perceberam-se neste estudo, diferenças entre os relatos dos cuidadores das instituições filantrópica e privadas, importantes na Qualidade do cuidado prestado ao idoso. Em relação à formação dos cuidadores, nas filantrópicas, muitos são admitidos para funções totalmente desvinculadas desta atividade, como por exemplo, ajudantes de obra. Estes profissionais, embora ascendidos à função de cuidador, muitas vezes continuam realizando as tarefas Que exerciam anteriormente. Assim, terminam por acumular funções restando-lhes pouco tempo para aprimorar-se e dedicar-se ao cuidado dos idosos. Mesmo assim, eles demonstram satisfação com a função, pois esta representa para muitos deles a possibilidade de inserção no mercado de trabalho. Considerando o aumento na demanda por instituições de longa permanência, como uma das conseQüências do envelhecimento populacional é necessário resgatar o sentido original de amparo e proteção da palavra (asylum), procurando tornar estes ambientes suportivos ao bem-estar e Qualidade de vida dos idosos. No momento em Que passam a coexistir instituições privadas, de caráter lucrativo, e filantrópicas é necessário pensar o papel da vigilância para a promoção da saúde nestes ambientes. A adeQuação das instituições filantrópicas as normas de funcionamento da Anvisa constitui um desafio para muitas destas. Serão as instituições filantrópicas capazes de reorganizar sua prática? Que suporte será necessário para Que as instituições possam tornar-se locais de amparo e proteção? Quais os limites da atuação do estado nas instituições?

\section{CONCLUSÃO}

Este estudo apontou problemas em relação à estrutura e a prestação de serviços a idosos nas instituições de longa permanência. Nestas instituições, especialmente as de caráter filantrópico, profissionais de serviços gerais, sem formação técnica, ao longo do tempo passam a atuar também como cuidadores de idosos, porém, sem receberem treinamento para esta atividade. Este Quadro indica a necessidade urgente de implementação das normas de funcionamento das instituições de longa permanência, enfaticamente nas filantrópicas.

\section{REFERÊNCIAS}

1. Souza ER, Minayo MCS, Ximenes LF, Deslantes SF. O idoso sob o olhar do outro. In: Minayo MCS, Coimbra Ir EA, organizadores. Antropologia, saúde e envelhecimento. Rio de Janeiro: Editora Fiocruz; 2002. p. 191-209.

2. Souza JAV, Freitas MC, Queiroz TA. Violência contra os idosos: análise documental. Rev Bras Enferm 2007; 60(3): 268-72.

3. Martinez SHL. O significado do cuidado para Quem cuida do idoso em uma instituição asilar [dissertação]. São Paulo: 
Universidade Federal de São Paulo; 2003.

4. Freitas CM. A vigilância da saúde para a promoção da saúde. In. Czeresnia D, Freitas CM. Promoção da saúde: conceitos, reflexões e tendências. Rio de Janeiro: Editora Fiocruz; 2004. p. 14I-59.

5. Buss PM. Promoção de saúde e Qualidade de vida. Ciênc saúde Coletiva 2000; 5(1): 163-77.

6. Born T, Boechat SN. A Qualidade dos cuidados ao idoso institucionalizado. In: Freitas EV, Py L, Cançado FAX, Doll I, Gorzoni ML, organizadores. Tratado de geriatria e gerontologia. Rio de Janeiro: Guanabara koogan; 2006. p. I I3 I-4I.

7. Donabedian A. Evaluating the Quality of medical care. Milbank Quaterly 2005; 83(4): 691-729.

8. Duarte MIRS. Cuidadores? Por Que e para Quê? Atenção ao idoso no domicílio. Rev Enferm 1996; 34:126-30.

9. Teixeira MH. A saúde do idoso: a arte de cuidar. Rio de laneiro: EDUERJ; 1998.

10. Ribeiro MTF, Ferreira RC, Magalhães CS, Ferreira EF, Moreira NA. Perfil dos cuidadores de idosos nas instituições de longa permanência de Belo Horizonte - MG. Ciênc Saúde Coletiva 2008; 13(4): 1285-92.

II. Brum AK, Tocantins FR, Silva, TJES. O enfermeiro como instrumento de ação no cuidar do idoso. Rev Latino-am Enfermagem 2005; 13(6): 1019-26.

12. Brêtas ACP. Cuidadores de idosos e o Sistema Único de Saúde. Rev Bras Enferm 2003; 56(3): 298-30I.

13. Ministério da Saúde (BR). Agência de Vigilância Sanitária. RDC $\mathrm{N}^{0} 283$ de 26 de setembro de 2005. Normas de funcionamento para as Instituições de Longa Permanência para Idosos [Internet].Brasília: Ministéiro da Saúde. [citado 8 jun 2009]. Disponível em: http://e-legis.anvisa.gov.br/leisref/public/
showAct.php

14. Ministério da Saúde (BR). Política Nacional de Saúde do Idoso - Portaria I395/GM [Internet]. Brasília: Ministério da Saúde [citado 8 jun 2009]. Disponível em: http://www.ufrgs.br/3idade/ portarial $395 \mathrm{gm} . \mathrm{html}$

15. Secretaria Municial da Saúde de Belo Horizonte. Portaria SMSA/SUS - BH no 052 de 15 de dezembro de 2000 [Internet]. Belo Horizonte: Secretaria Municipal de Saúde. [citado 3 jan 2006]. Disponível em: http://www.pbh.gov.br

16. Néri AL, Sommerhalder C. Cuidar de idosos no contexto da família: Questões psicológicas e sociais. Campinas: Alínea Editora; 2001

17. Beauvoir S. A velhice. Rio de Janeiro: Nova Fronteira; 1990.

18. Cunha UGV, Barros Jr. OR, Siqueira AL. Levantamento epidemiológico psicogeriátrico em asilos. I Bras Psieuiatr 1985; 34(6): 389-94.

19. Silva EBN. A Instituição e o idoso: um estudo das características da instituição e do perfil de seus moradores. Gerontol 1998; 6(4): 167-76.

20. Telles Filho ICP, Petrilli Filho JF. Causas de inserção de idosos em uma instituição asilar. Esc Anna Nery Rev Enferm 2002; 6(1): 135-43.

21. Organisation for Economic Co-operation and Development. Directorate for employment, labour and social affairs health committee. Policies for health ageing: an overview. Paris: OECD; 2009.

22. Mazo GZ, Benedetti TB. Condições de vida dos idosos institucionalizados na Grande Florianópolis. Rev Ciência Saúde 1999; 18(1/2):51-6.

23. Chaimowicz F, Greco Dinâmica da institucionalização de idosos em Belo horizonte, Brasil. Rev Saúde Pública 1999; 33(5): 454-60. 